\title{
OFF-AXIS AFTERGLOW EMISSION FROM JETTED GAMMA-RAY BURSTS
}

\author{
Jonathan Granot, ${ }^{1}$ Alin Panaitescu, ${ }^{2}$ Pawan Kumar, ${ }^{1}$ and Stan E. Woosley ${ }^{3}$ \\ Received 2002 January 21; accepted 2002 April 2; published 2002 April 17
}

\begin{abstract}
We calculate gamma-ray burst (GRB) afterglow light curves from a relativistic jet as seen by observers at various viewing angles, $\theta_{\mathrm{obs}}$, relative to the jet axis. We describe three increasingly more realistic models and compare the resulting light curves. An observer at $\theta_{\text {obs }}<\theta_{0}$, where $\theta_{0}$ is the initial jet opening angle, should see a light curve very similar to that for an on-axis observer. An observer at $\theta_{\text {obs }}>\theta_{0}$ sees a rising light curve at early times, peaking when the jet Lorentz factor is $\sim 1 / \theta_{\text {obs }}$, and approaching that seen by an on-axis observer, at later times. A strong linear polarization $(\$ 40 \%)$ may occur near the peak in the light curve and slowly decay with time. We show that, if GRB jets have a universal energy, then orphan afterglows are detectable up to a maximum offset angle that is independent of the jet initial aperture and thus at a rate proportional to the true GRB rate. We also discuss the implications of the proposed connection between SN 1998bw and GRB 980425.
\end{abstract} Subject headings: gamma rays: bursts — ISM: jets and outflows — radiation mechanisms: nonthermal

\section{INTRODUCTION}

Gamma-ray bursts (GRBs) are explosions that release roughly $10^{51}$ ergs as kinetic energy of highly relativistic material (Frail et al. 2001; Panaitescu \& Kumar 2001). The achromatic break of the afterglow 990510 (Harrison et al. 1999; Stanek et al. 1999) and the breaks seen in the optical emission of several other afterglows show that some GRBs are highly nonspherical (Rhoads 1999). If we are not located within the initial jet aperture $\theta_{0}$, the GRB and early afterglow are very weak owing to the relativistic beaming of photons away from our line of sight. As the jet decelerates, the relativistic beaming becomes less severe and the emission from the jet becomes detectable to observers at larger viewing angles $\theta_{\text {obs }}$. Granot et al. (2001) have shown that the light curve seen by an observer located within the initial jet aperture $\left(\theta_{\mathrm{obs}}<\theta_{0}\right)$ is very similar to that for an on-axis observer $\left(\theta_{\mathrm{obs}}=0\right)$. Dalal, Griest, \& Pruet (2002) and Rossi, Lazzati, \& Rees (2002) have presented simple models to calculate the flux in this case. We reanalyze these models in $\S 2.1$ and consider more realistic models in $\S \S 2.2$ and 2.3. Moderski, Sikora, \& Bulik (2000) have calculated off-axis light curves with a more complex model, similar to that presented in $\S 2.2$. In $\S 3$, we calculate the temporal evolution of the linear polarization for various $\theta_{\text {obs }}$. The prospects of using the detection rate of orphan afterglows to estimate the collimation of GRB jets are analyzed in $\S 4$. In $\S 5$, we analyze the suggestion of Woosley, Eastman, \& Schmidt (1999) that a relativistic jet emanating from the supernova (SN) explosion and pointing away from us could explain the observations of SN 1998bw and GRB 980425.

\section{CALCULATION OF AFTERGLOW EMISSION FOR OFF-AXIS OBSERVERS}

For simplicity, we consider in this section only jets propagating into a homogeneous medium. To improve our understanding of the results and to assess how robust they are, we consider three models of increasing complexity.

\footnotetext{
${ }^{1}$ Institute for Advanced Study, Olden Lane, Princeton, NJ 08540.

${ }^{2}$ Department of Astrophysical Sciences, Princeton University, Peyton Hall, Ivy Lane, Princeton, NJ 08544.

${ }^{3}$ Department of Astronomy and Astrophysics, University of California at Santa Cruz, 477 Clark Kerr Hall, Santa Cruz, CA 95064.
}

\subsection{Model 1: A Point Source on the Jet Axis}

We begin with a simple model where we assume that the afterglow synchrotron emission arises from a point moving along the jet axis and whose dynamics is that calculated by Rhoads (1999): $\gamma=\theta_{0}^{-1}\left(t_{0} / t_{\mathrm{jet}}\right)^{-3 / 8}$ at $t_{0}<t_{\mathrm{jet}}$ and $\gamma=\theta_{0}^{-1}\left(t_{0} / t_{\mathrm{jet}}\right)^{-1 / 2}$ at $t_{0}>t_{\text {jet }}$, where $t_{\text {jet }}=6.2(1+z)\left(E_{52} / n_{0}\right)^{1 / 3}\left(\theta_{0} / 0.1\right)^{8 / 3} \mathrm{hr}$ (Sari, Piran, \& Halpern 1999) is the "jet break time" measured by an on-axis observer, $E_{52}$ being the isotropic equivalent energy in units of $10^{52} \mathrm{ergs}, n_{0}$ the ambient density in $\mathrm{cm}^{-3}$, and $z$ the GRB redshift. For $\theta_{\text {obs }}=0$, the light curve is that calculated analytically by Sari, Piran, \& Narayan (1998), Rhoads (1999), and Sari et al. (1999). For a point source moving at angle $\theta$ relative to the observer, the observed flux is

$$
F_{\nu}=\frac{L_{\nu^{\prime}}^{\prime}}{4 \pi d_{A}^{2}}\left(\frac{\nu}{\nu^{\prime}}\right)^{3}=\frac{1+z}{4 \pi d_{L}^{2}} \frac{L_{\nu^{\prime}}^{\prime}}{\gamma^{3}(1-\beta \cos \theta)^{3}},
$$

where $d_{L}$ is the luminosity distance, $L_{\nu^{\prime}}^{\prime}$ and $\nu^{\prime}$ are the jet comoving frame spectral luminosity and frequency, and $\gamma=$ $\left(1-\beta^{2}\right)^{-1 / 2}$ is the jet Lorentz factor. Let $t$ and $\nu$ be the observed time and frequency for an observer at $\theta$, and $t_{0}$ and $\nu_{0}$ those for an on-axis observer. Since $t / t^{\prime} \approx d t / d t^{\prime}=\nu^{\prime} / \nu=(1+$ z) $\gamma(1-\beta \cos \theta)$, we obtain

$$
\frac{t_{0}}{t}=\frac{\nu}{\nu_{0}}=\frac{(1-\beta)}{(1-\beta \cos \theta)} \equiv a \approx \frac{1}{\left(1+\gamma^{2} \theta^{2}\right)},
$$

$$
F_{\nu}\left(\theta_{\mathrm{obs}}, t\right)=a^{3} F_{\nu / a}(0, a t)
$$

The light curves obtained using equation (3) are shown with dashed lines in Figure 1. At first, $\gamma \theta_{\text {obs }} \gg 1$ and $a^{3} \approx\left(\gamma \theta_{\text {obs }}\right)^{-6}$ is the dominant term in equation (3), giving a sharp rise in the light curve. The afterglow emission peaks when $\gamma \sim 1 / \theta_{\text {obs }}$, after which the flux begins to decay, asymptotically approaching the on-axis light curve.

The main advantage of model 1 is that it is very simple, and nevertheless gives reasonable results for $\theta_{\text {obs }} \gtrsim 2 \theta_{0}$. Its main drawback is that it is not physical for $\theta_{\text {obs }} \lesssim \theta_{0}$ at $t \lesssim t_{\text {jet }}$. For $\theta_{\text {obs }}<\theta_{0}$, the observed radiation is initially dominated by emission from within an angle $1 / \gamma<\theta_{\text {obs }}$ around the line of sight, while in model 1 the emission is always only from the jet axis; 


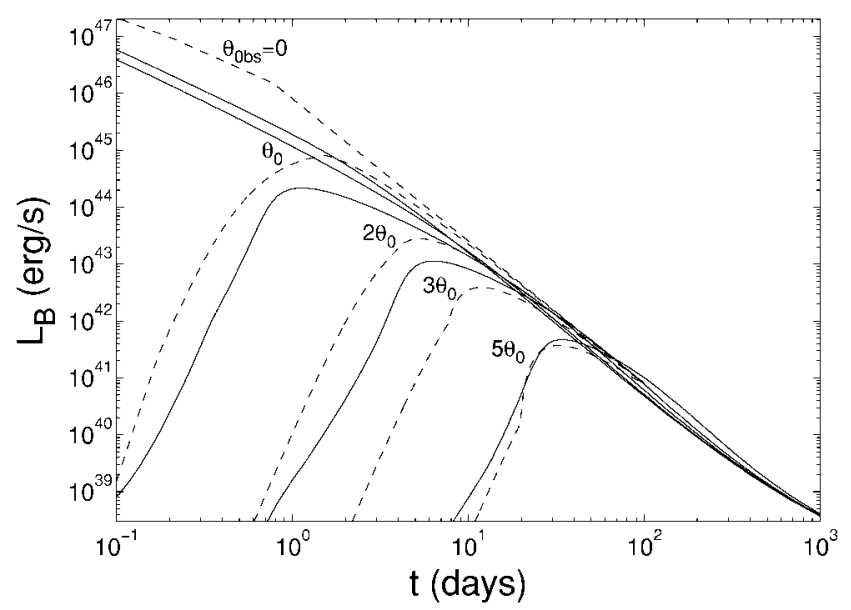

FIG. 1. $-B$-band luminosity for models 1 (dashed lines) and 2 (solid lines) for $\theta_{0}=5^{\circ}, \theta_{\mathrm{obs}}=(0,1,2,3,5) \theta_{0}, E_{52}=80, n_{0}=1, p=2.5, \epsilon_{B}=0.01$, and $\epsilon_{e}=0.1$, where $\epsilon_{B}$ and $\epsilon_{e}$ are the fraction of the internal energy in the magnetic field and electrons, respectively, and $p$ is the power-law index of the electron energy distribution. Note that model 1 is scaled down by a factor of 2.5 to help compare between the two models.

therefore, the dominant contribution to the emission is missing until the time when $\gamma \sim 1 / \theta_{\text {obs }}$. This problem is overcome by our next model.

\subsection{Model 2: A Homogeneous Jet}

This model is described in Kumar \& Panaitescu (2000). The Lorentz factor and energy per solid angle are considered independent of $\theta$ within the jet aperture. The jet deceleration is calculated from the mass and energy conservation equations, and the jet expands laterally at the local sound speed. The calculation of radiative losses includes synchrotron and inverse Compton, and the synchrotron spectrum is taken to be a piecewise power law with the usual self-absorption, cooling, and injection break frequencies, calculated from the cooled electron distribution and magnetic field. The observed flux is obtained by integrating the jet emission over the equal arrival time surface.

The light curves of model 2 are shown with solid lines in Figure 1. The flux density in the decaying stage (when the entire jet is visible) increases slightly with $\theta_{\text {obs }}$ because, for a given observer time, the emission received at larger $\theta_{\text {obs }}$ arises at smaller radii, when the jet is intrinsically brighter. At a few hundred days, the light curves begin to flatten owing to the transition to the nonrelativistic regime.

The light curves for $\theta_{\text {obs }} \lesssim \theta_{0}$ are very different from those of model 1 (and more realistic). Furthermore, the light curves for $\theta_{\mathrm{obs}} \leq \theta_{0}$ are very similar to $\theta_{\mathrm{obs}}=0$ in this model. Since the jet is homogeneous, the ratio of fluxes for $\theta_{\mathrm{obs}}<\theta_{0}$ and $\theta_{\text {obs }}=0$ is the ratio $\left(>\frac{1}{2}\right)$ of the areas within the jet opening that subtend an angle of $1 / \gamma$ around these directions.

We note that the light curves of model 1 for $\theta_{\text {obs }} / \theta_{0}=$ 1,2 are much closer to the light curves of model 2 for $\theta_{\text {obs }} / \theta_{0}=2,3$, respectively, than to the model 2 light curves for the same viewing angles, because the emission received at $\theta_{\text {obs }}>\theta_{0}$ is dominated by the region on the jet surface that is closest to the direction toward the observer. Therefore, model 1 becomes more accurate if $\theta=\max \left(0, \theta_{\text {obs }}-\theta_{0}\right)$ is used instead of $\theta=\theta_{\text {obs }}$ in equations (1) and (2).

The main advantage of model 2 is that it provides more realistic light curves with a very small computational effort,

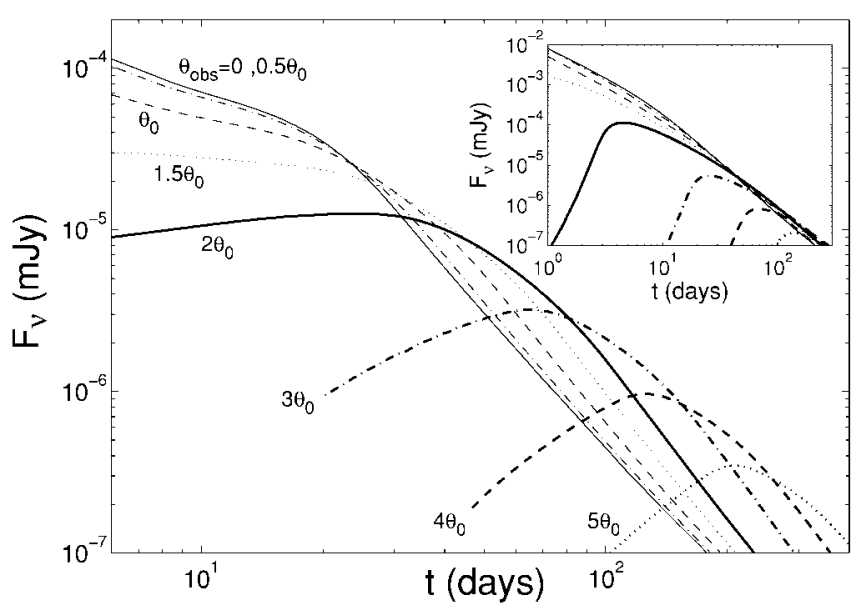

FIG. 2.-Light curves of model 3 for $\theta_{0}=0.2, E_{52}=n_{0}=z=1, p=$ $2.5, \epsilon_{e}=0.1, \epsilon_{B}=0.01$, and $\nu=5 \times 10^{14} \mathrm{~Hz}$. The inset shows the same light curves for model 2 , where the same traces correspond to the same viewing angles $\theta_{\text {obs }}$.

making it convenient to use for data fitting (e.g., Panaitescu \& Kumar 2001). Its main drawback is the simplified treatment of the dynamics, which leads to some differences relative to our next model.

\subsection{Model 3: Two-Dimensional Hydrodynamical Simulation}

This model is described in Granot et al. (2001). The jet dynamics is obtained with a two-dimensional hydrodynamical simulation, with initial conditions of a wedge taken from the spherical self-similar Blandford-McKee (1976) solution. The afterglow light curves are calculated considering the emission from all the shocked region, taking into account the relativistic transformations of the radiation field, and the different photon arrival times to the different observers.

Figure 2 shows the light curves of model 3, while the inset provides the light curves of model 2 for the same set of parameters. In model 3 , the peak of the light curves for $\theta_{\mathrm{obs}}>$ $\theta_{0}$ is flatter compared to model 2 and occurs at a somewhat later time. The rise before the peak is not as sharp as in models 1 or 2 , since in model 3 there is some material at the sides of the jet with a moderate Lorentz factor (Granot et al. 2001; Piran \& Granot 2001), whose emission dominates the observed flux at early times for $\theta_{\mathrm{obs}}>\theta_{0}$. The light curves for $\theta_{\mathrm{obs}}>\theta_{0}$ peak at a later time compared to model 2 , and the flux during the decay stage grows faster with $\theta_{\text {obs }}$, because in model 3 the curvature of the shock front is larger and the emission arises within a shell of finite width, so that smaller radii contribute to a given observer time. The light curves for models 2 and 3 are quantitatively similar for $\theta_{\mathrm{obs}}<\theta_{0}$.

The main advantage of this model is a reliable and rigorous treatment of the jet dynamics, which provides insight on the behavior of the jet and the corresponding light curves. Its main drawback is the long computational time it requires.

\section{LINEAR POLARIZATION}

While a spherical afterglow should exhibit little or no linear polarization, as the polarizations from the different parts of the afterglow image cancel out, a jetted afterglow breaks the circular symmetry of the afterglow image for $\theta_{\text {obs }}>0$ and may have a polarization of $\leqslant 20 \%$ for $\theta_{\mathrm{obs}}<\theta_{0}$ (Ghisellini \& Lazzati 1999; Sari 1999). One might therefore expect an even larger polari- 
zation for $\theta_{\text {obs }}>\theta_{0}$. An isotropic magnetic field configuration in the local rest frame will produce no linear polarization. However, as the magnetic field is most likely produced at the shock itself, one might expect the magnetic field perpendicular $\left(B_{\perp}\right)$ and parallel $\left(B_{\|}\right)$to the shock direction to have different magnitudes (Gruzinov 1999; Sari 1999). We calculate the linear polarization for model 2 following Ghisellini \& Lazzati (1999) ${ }^{4}$ and using their notations. They assume the magnetic field is strictly in the plane of the shock $\left(B=B_{\perp}\right)$.

Figure 3 shows the polarization as a function of time for different $\theta_{\mathrm{obs}}$ in terms of $P_{60}$. For $P_{60}<0$ the polarization is along the plane containing the line of sight and the jet axis, while for $P_{60}>0$ it is rotated by $90^{\circ}$ (for $\left\langle B_{\perp}\right\rangle<2\left\langle B_{\|}\right\rangle$this is reversed; e.g., Sari 1999). A more isotropic magnetic field configuration would result in a smaller degree of polarization, so the value of the polarization in Figure $3(\$ 40 \%)$ may be viewed as a rough upper limit. For $0.3 \leqq \theta_{\text {obs }} / \theta_{0} \lesssim 1.1$, the polarization vanishes and reappears rotated by $90^{\circ}$ around $t_{\text {jet }}$. This behavior may occur again at a later time, but the subsequent polarization is very low. For $1.1 \lesssim \theta_{\text {obs }} / \theta_{0} \lesssim 1.6$, the polarization has two peaks, the first higher than the second. For $\theta_{\mathrm{obs}} / \theta_{0} \gtrsim 1.1$, the polarization is largest near the peak in the light curve and decreases quite slowly with time, while the maximum polarization shows a very weak dependence on $\theta_{\text {obs }}$ and is about a factor of 2 larger than for $\theta_{\mathrm{obs}}=\theta_{0}$.

\section{ORPHAN AFTERGLOWS}

If GRB jets have well-defined edges, the prompt gammaray flux drops very sharply outside the opening of the jet, and the prompt burst will be very hard to detect from $\theta_{\mathrm{obs}}>\theta_{0}$. On the other hand, the afterglow emission may be detected out to a larger angle, $\theta_{\text {det }}>\theta_{0}$, where the exact value of $\theta_{\text {det }}$ depends on the jet parameters (including its redshift), the observed band, and the limiting flux for detection. Jetted GRBs with $\theta_{0}<$ $\theta_{\text {obs }}<\theta_{\text {det }}$ are expected to be orphan afterglows (i.e., detectable in the optical but not in gamma rays).

It has been argued by Dalal et al. (2002) that $\theta_{\text {det }} / \theta_{0} \approx$ const for $\theta_{0} \ll 1$, so that the detection rate of orphan afterglows $\dot{N}_{\text {orph }}^{\text {det }}$ will be a constant [namely, $\left(\theta_{\text {det }} / \theta_{0}\right)^{2}$ ] times the GRB detection rate $\dot{N}_{\mathrm{GRB}}^{\mathrm{det}}$, and thereby a comparison between these two rates will not constrain $\theta_{0}$ or the true rate of GRBs $\dot{N}_{\mathrm{GRB}}^{\text {true }}$. This result was obtained assuming a constant flux, $F_{\nu}\left(t_{\text {jet }}\right)$, at $t_{\text {jet }}$ for $\theta_{\mathrm{obs}}=0$. However, afterglow observations suggest that the total energy in the jet, $E_{\text {jet }}$, is roughly constant (Frail et al. 2001; Panaitescu \& Kumar 2001; Piran et al. 2001), while $F_{\nu}\left(t_{\text {jet }}\right)$ varies over a wider range. In fact, for $E_{\text {jet }}=$ const, simple jet models (Rhoads 1999; Sari et al. 1999) predict that the hydrodynamical evolution of the jet (and therefore the light curves for all $\theta_{\mathrm{obs}}$ ) becomes independent of $\theta_{0}$ once the jet enters the phase of exponential lateral expansion with radius. This corresponds to $t>t_{\text {jet }}$ for $\theta_{\mathrm{obs}}<\theta_{0}$ and to $t \gtrsim\left(\theta_{\text {obs }} / \theta_{0}\right)^{2} t_{\text {jet }}$ for $\theta_{\text {obs }}>\theta_{0}$, which includes the time around or after the peak in the light curve. This implies that for $E_{\text {jet }}=$ const, we have $\theta_{\mathrm{det}}=$ const $\equiv \theta_{\mathrm{det}, 0}$ (rather than $\theta_{\operatorname{det}} / \theta_{0}=$ const) for $\theta_{0}<\theta_{\text {det }, 0}$. For $\theta_{0} \geqslant \theta_{\text {det }, 0}$, naturally $\theta_{\text {det }}$ is larger than $\theta_{0}$ if the afterglow is detectable from $\theta_{\text {obs }}<\theta_{0}$, but the solid angle between $\theta_{0}$ and $\theta_{\text {det }}, \Omega_{\text {orph }}$, where $\Omega_{\text {orph }} / 4 \pi=\cos \theta_{0}-\cos \theta_{\text {det }} \approx$ $\left(\theta_{\mathrm{det}}^{2}-\theta_{0}^{2}\right) / 2$ (in which we have detectable orphan afterglows), remains approximately constant. Furthermore, the distribution of $\theta_{0}$ inferred from observations (Frail et al. 2001; Panaitescu \&

\footnotetext{
${ }^{4}$ In contrast with the calculation of Ghisellini \& Lazzati (1999), we include lateral expansion of the jet and integrate over the equal photon arrival time surfaces, which leads to some differences in the results for $\theta_{\text {obs }}<\theta_{0}$.
}

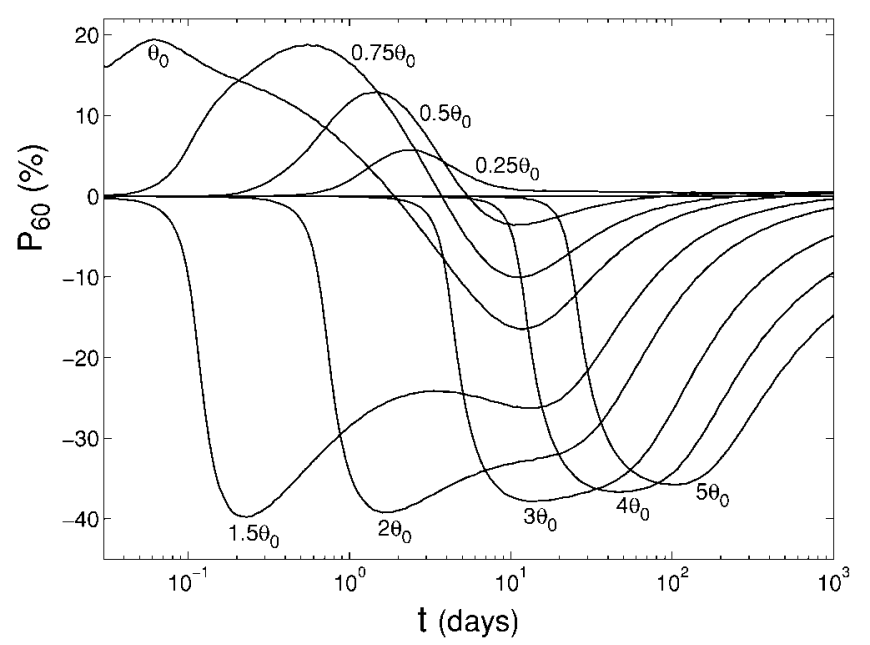

FIG. 3.-Linear polarization for model 2 for several viewing angles and for the same parameters as in Fig. 1.

Kumar 2001) is sharply peaked at low $\theta_{0}\left(\sim 2^{\circ}-3^{\circ}\right)$. This suggests that most of the orphan afterglows that would be detected should have $\theta_{0} \sim 3^{\circ}$. For a reasonable limiting magnitude for detection, this implies $\theta_{\text {det }} \gg \theta_{0}$ in most cases, and therefore $\theta_{\text {det }} \approx \theta_{\text {det }, 0}$. For example, for model 2 with $E_{\text {jet }}=5 \times 10^{50}$ ergs (assuming a double-sided jet), $\epsilon_{e}=0.1, \epsilon_{B}=0.01, p=2.5, n_{0}=z=1$, and a limiting magnitude for detection of $R=24$, we obtain $12.4<\theta_{\text {det }}<23^{\circ}$ and $0.023<\Omega_{\text {orph }} / 4 \pi<0.045$ for $2^{\circ}<\theta_{0}<$ $15^{\circ}$. If indeed $\Omega_{\text {orph }} \approx$ const, then $N_{\text {orph }}^{\text {det }}$ should provide a good estimate of the true GRB rate, $\dot{N}_{\text {GRB }}^{\text {true }} \stackrel{=}{=}\left(4 \pi / \Omega_{\text {orph }}\right) \dot{N}_{\text {orph }}^{\text {det }}$. The average beaming fraction $f_{b}=\left\langle 1-\cos \theta_{0}\right\rangle \approx\left\langle\theta_{0}^{2}\right\rangle / 2$ is given by $f_{b}=\dot{N}_{\mathrm{GRB}}^{\mathrm{det}} / \dot{N}_{\mathrm{GRB}}^{\text {true }}=\left(\Omega_{\text {orph }} / 4 \pi\right) \dot{N}_{\mathrm{GRB}}^{\mathrm{det}} / \dot{N}_{\mathrm{orph}}^{\mathrm{det}}$.

\section{GRB $980425 / \mathrm{SN} 1998 \mathrm{bw}$}

On 1998 April 25, a GRB was detected by BeppoSAX and the Compton Gamma Ray Observatory. The burst consisted of a single wide peak of duration $30 \mathrm{~s}$, peak flux in the $24-1820 \mathrm{keV}$ band of $3 \times 10^{-7} \mathrm{ergs} \mathrm{cm}^{-2} \mathrm{~s}^{-1}$, and fluence of $4.4 \times 10^{-6} \mathrm{ergs} \mathrm{cm}^{-2}$ (Soffitta et al. 1998; Kippen et al. 1998). The burst had no detectable emission above $300 \mathrm{keV}$. The burst spectrum was a broken power law with the break at $148 \pm 33 \mathrm{keV}$ and the high-energy power-law photon index of $-3.8 \pm 0.7$ (see Galama et al. 1998). These values are not unusual for GRBs.

A bright SN Ic, SN 1998bw, located at $z=0.0085$, was detected within $8^{\prime}$ of GRB 980425 . From the extrapolation of optical light curves, Galama et al. (1998) suggested that the SN went off within a day of the GRB, thereby implying a possible connection between the two events. The probability of this association is strengthened by the uniquely peculiar light curve and spectrum of the SN (e.g., Patat et al. 2001). Early on, Woosley et al. (1999) gave arguments why SN 1998bw might be an SN exploded by a jet and therefore possibly associated with a GRB. This would arise, for instance, in the collapsar model (Woosley 1993; MacFadyen \& Woosley 1999; Paczyński 1998).

If indeed the two events are associated, then the total isotropic equivalent of energy release in gamma rays for GRB 980425 is $E_{\gamma \text {, iso }}=8.5 \times 10^{47} \mathrm{ergs}$, or a factor of $\sim 10^{4}$ smaller than the energy for an average cosmological GRB. This could be explained in two ways.

A sharp-edged, homogeneous jet seen at $\theta_{\mathrm{obs}}>\theta_{0}$.- If GRB 980425 was viewed at $\theta_{\mathrm{obs}}>\theta_{0}$, it might explain its small $E_{\gamma, \text { iso }}$. For a GRB arising from a jet with $\gamma$ independent of $\theta$ 
and sharp edges, the observed energy falls off rapidly for $\theta_{\text {obs }}>\theta_{0}$, in fact, as $b^{6}$, where $b \equiv \gamma\left(\theta_{\text {obs }}-\theta_{0}\right)$. Moreover, for an observer at $\theta_{\text {obs }}>\theta_{0}$, the peak of the spectrum is lower by a factor of $b^{2}$ and the burst duration longer by the same factor, compared to an observer at $\theta_{\text {obs }}<\theta_{0}$. Therefore, the low $E_{\gamma \text {, iso }}$ of 980425 implies $b^{6} \sim 10^{4}$ and $\theta_{\text {obs }} \sim \theta_{0}+5^{\circ}(\gamma / 50)^{-1}$. If GRB 980425 were at a cosmological distance and seen from $\theta_{\text {obs }}<$ $\theta_{0}$, the peak of the spectrum and the burst duration would be $\sim 1[3 /(1+z)] \mathrm{MeV}$ and $4[(1+z) / 3] \mathrm{s}$, respectively.

A second constraint is set by the condition that the optical afterglow is dimmer than SN 1998bw, which had a luminosity of $2 \times 10^{42}$ ergs at 1 day, rose to $10^{43}$ ergs at 14-19 days (Galama et al. 1998), then decayed at $\sim 0.017 \mathrm{mag} \mathrm{day}^{-1}$ (Patat et al. 2001). To compare it with the afterglow luminosity, we shall use the "typical" properties of the afterglows whose optical light curves exhibited breaks: (1) average jet break time $t_{\text {iet }} \sim 0.5$ days in their rest frame, (2) average optical luminosity $L\left(\theta_{\text {obs }}=0, t_{\text {jet }}\right) \sim 2 \times 10^{45} \mathrm{ergs} \mathrm{s}^{-1}$ at $t_{\text {jet }}$, (3) $L \propto t^{-\alpha}$ with $\alpha \sim 2$ at $t>t_{\text {jet }}$, and (4) $L_{\nu} \propto \nu^{-\beta}$, with $\beta \sim 1$, at optical frequencies.

Using model 1 described in $\S 2.1$, it can be shown that for an observer at $\theta_{\text {obs }} \gtrsim 2 \theta_{0}$ the time and optical luminosity at the light curve peak are

$$
t_{p} \simeq(5+2 \ln \Theta) \Theta^{2} t_{\mathrm{jet}}, \quad L_{p} \simeq 2^{-(\beta+3)} \Theta^{-2 \alpha} L\left(0, t_{\mathrm{jet}}\right),
$$

where $\Theta \equiv\left(\theta_{\text {obs }} / \theta_{0}\right)-1$. From these equations, it can be shown that for $\theta_{\text {obs }} \geqslant 3 \theta_{0}$ the peak afterglow luminosity $L_{p}\left(t_{p}\right)$ is a factor of $\sim 3$ lower than $L(t)$ of SN 1998bw. During the decay phase, the afterglow luminosity remains below that of the $\mathrm{SN}$ until about 400 days, when they become comparable. We note that Patat et al. (2001) report a flattening of the SN 1998bw decay after 375 days, to a dimming rate of $\sim 0.009 \mathrm{mag} \mathrm{day}^{-1}$, which they interpret as the settling in of the ${ }^{56} \mathrm{Co}$ decay or the existence of other sources. Together with the above constraint, $\theta_{\mathrm{obs}} \sim \theta_{0}+$ $5^{\circ}(\gamma / 50)^{-1}$, imposed by the fluence of GRB 980425 , the condition that the afterglow emission does not exceed that of $\mathrm{SN}$ 1998 bw leads to $\theta_{0} \lesssim 3^{\circ}(\gamma / 50)^{-1}$ and $3 \theta_{0} \lesssim \theta_{\text {obs }} \leqslant 8^{\circ}(\gamma / 50)^{-1}$.

Inhomogeneous jet seen off-axis.-Another possibility is that the jet does not have sharp edges but wings of lower energy and Lorentz factor that extend to large $\theta$. Such a picture of the jet was suggested by Woosley et al. (1999) and is consistent with the relativistic studies of the collapsar model by W. Zhang, S. E. Woosley, \& A. MacFadyen (2002, in preparation). GRB 980425 would be then be produced by material with $\gamma \sim 10$ moving in our direction.
When seen on-axis, a "typical" afterglow has $L \sim 5 \times$ $10^{44} \mathrm{ergs} \mathrm{s}^{-1}$ at 1 day and $L \sim 2 \times 10^{42} \mathrm{ergs} \mathrm{s}^{-1}$ at 16 days; i.e., it would be 200 times brighter than SN 1998bw when its first observation was made and several times dimmer when SN 1998bw peaked. All other parameters remaining the same, an afterglow emission dimmer than that of SN 1998bw at 1 day requires an energy per solid angle $\epsilon\left(\theta_{\text {obs }}\right)$ in the direction toward the observer satisfying $\epsilon\left(\theta_{\mathrm{obs}}\right) \theta_{\mathrm{obs}}^{2} \sim(200)^{-3 /(p+3)} E_{\mathrm{jet}} \sim 2 \times 10^{49}$ ergs. As in the previous subsection, the observer location should satisfy $\theta_{\text {obs }} \geqslant 3 \theta_{0}$ so that the optical emission of the main jet of opening angle $\theta_{0}$ is below the $\mathrm{SN}$ light curve.

\section{CONCLUSION}

We have presented light curves of jetted GRB afterglows for arbitrary observer locations, obtained with three models of increasing complexity: a point source moving along the jet axis (§ 2.1), a uniform jet ( $\$ 2.2$ ), and a two-dimensional hydrodynamical simulation $(\S 2.3)$. The basic features of the light curves for $\theta_{\text {obs }}>\theta_{0}$ are similar in all models. Moreover, the uniform jet model 2 (§ 2.2) is in rough quantitative agreement with the hydrodynamical model.

We find that if one assumes a universal jet energy, orphan optical afterglows (associated with off-axis jets) can be detected up to a constant $\theta_{\text {obs }}$ rather than a constant $\theta_{\text {obs }} / \theta_{0}$, as suggested by Dalal et al. (2002). Therefore, future searches of orphan afterglows based on their rising light curves, power-law optical spectra (which may soften around the time of the peak $t_{p}$, for $t_{p} \lesssim$ a few days, while the spectral index $\beta>0$ at $t \gtrsim t_{p}$ ), and large degree of linear polarization $(\Sigma 40 \%)$ may constrain the distribution of $\theta_{0}$ and the true GRB rate, providing a new line of evidence in favor of jetted GRB outflows. Recently, Huang, Dai, \& Lu (2002) have suggested another scenario (failed GRBs) for producing orphan afterglows, which would increase their detection rate. A good afterglow monitoring can distinguish between the rising light curves of jets seen at $\theta_{\mathrm{obs}}>\theta_{0}$ and the monotonically dimming light curves of afterglows from failed GRBs.

We thank Mark Miller for generating the data for model 3. This research was supported by NSF grant PHY 99-79985 and MCA93S025 (computational support), NSF grant PHY 0070928 (J. G.), a Lyman Spitzer, Jr., Fellowship (A. P.), and grants NAG5-8128 and MIT-292701 (S. W.).

\section{REFERENCES}

Blandford, R. D., \& McKee, C. F. 1976, Phys. Fluids, 19, 1130

Dalal, N., Griest, K., \& Pruet, J. 2002, ApJ, 564, 209

Frail, D., et al. 2001, ApJ, 562, L55

Galama, T., et al. 1998, Nature, 395, 670

Ghisellini, G., \& Lazzati, D. 1999, MNRAS, 309, L7

Granot, J., et al. 2001, in GRBs in the Afterglow Era, ed. E. Costa, F. Frontera, \& J. Hjorth (Berlin: Springer), 312

Gruzinov, A. 1999, ApJ, 525, L29

Harrison, F., et al. 1999, ApJ, 523, L121

Huang, Y., Dai, Z., \& Lu, T. 2002, MNRAS, in press (astro-ph/0112469)

Kippen, R., et al. 1998, GCN Circ. 67 (http://gcn.gsfc.nasa.gov/gcn/gen3/ 067.gcn3)

Kumar, P., \& Panaitescu, A. 2000, ApJ, 541, L9

MacFadyen, A., \& Woosley, S. 1999, ApJ, 524, 262

Moderski, R., Sikora, M., \& Bulik, T. 2000, ApJ, 529, 151

Paczyński, B. 1998, ApJ, 494, L45
Panaitescu, A., \& Kumar, P. 2001, ApJ, 560, L49

Patat, F., et al. 2001, ApJ, 555, 900

Piran, T., \& Granot, J. 2001, in GRBs in the Afterglow Era, ed. E. Costa, F. Frontera, \& J. Hjorth (Berlin: Springer), 300

Piran, T., Kumar, P., Panaitescu, A., \& Piro, L. 2001, ApJ, 560, L167

Rhoads, J. 1999, ApJ, 525, 737

Rossi, E., Lazzati, D., \& Rees, M. J. 2002, MNRAS, in press (astro-ph/ 0112083)

Sari, R. 1999, ApJ, 524, L43

Sari, R., Piran, T., \& Halpern, J. 1999, ApJ, 519, L17

Sari, R., Piran, T., \& Narayan, R. 1998, ApJ, 497, L17

Soffitta, P., et al. 1998, IAU Circ. 6884

Stanek, K., et al. 1999, ApJ, 522, L39

Woosley, S. E. 1993, ApJ, 405, 273

Woosley, S. E., Eastman, R., \& Schmidt, B. 1999, ApJ, 516, 788 